\title{
Common Misconceptions about the Galactic Cluster Calibration of the Cepheid PLC Relation
}

\author{
David G. Turner \\ Saint Mary's University, Halifax, Nova Scotia, B3H 3C3, Canada
}

Comments in the literature in recent years reveal a variety of misconceptions about the role and value of galactic clusters for calibrating the Cepheid period-luminosity-colour (PLC) relation. This paper attempts to clarify some of these points using personal experiences gained from studies of the sparse galactic clusters and groups in which some classical Cepheids are located.

\section{Interstellar reddening}

Reddening is often blamed for problems originating from other causes. Three features of reddening are sometimes overlooked: (i) no universal reddening law applies to all fields, (ii) mean reddening laws are not applicable to samples of dispersed objects, and (iii) patchy extinction is the norm.

The effect of ignoring such points can be seen in the precision of older estimates of distance modulus for the Cepheid calibrating clusters. For clusters reddened by $E_{B-V} \approx 0.5$, a spread in reddening slopes $E_{U-B} / E_{B-V}$ for cluster $\mathrm{B}$-stars from 0.65 to 0.85 (typical of the galactic plane) results in a dispersion of \pm 0.19 in distance modulus if they are dereddened using a common reddening law. Typical uncertainties in zero-age main-sequence (ZAMS) fits under such conditions are probably no better than \pm 0.15 , while the colour spread of individual Cepheids in the instability strip accounts for an additional scatter of \pm 0.08 (or more). The expected scatter in the Cepheid period-luminosity (PL) relation amounts to \pm 0.25 , typical of what is observed (Gieren \& Fouque 1993). Similar techniques used with Strömgren photometry produce no better results. Adoption of individual reddening corrections for cluster stars and use of reddening laws specific for each field can reduce the dispersion in cluster distance moduli to \pm 0.05 or less. With dispersion in the instability strip included, the scatter in the Cepheid PL relation should be no larger than about \pm 0.09 . Gieren \& Fouqué (1993) expressed surprise that the dispersion for cluster calibrators from the author's program should be so small, but it is exactly the result expected from studying clusters in a detailed, homogeneous fashion. Recent results from the program have also led to an estimate of $2.1 \pm 0.2$ for the colour term of the PLC relation from galactic calibrators, consistent with values obtained for Cepheids in Magellanic Cloud clusters. Metallicity spread in the calibrating clusters is apparently not an obvious problem - yet.

Variations in the reddening law also affect the delineation of the Cepheid instability strip. For example, Fernie's (1990a) photometric reddenings for bright Cepheids are a poor match to space reddenings obtained for the same variables; photometric colour excesses for 13 Cepheids with reliable space reddenings differ in the mean (space - photometric) by $\Delta E_{B-V}=-0.01 \pm 0.06$ s.d. The scatter is large, being largest for the longest period Cepheids. The wedge-shaped instability strip delineated by Fernie (1990b) using photometric reddenings con- 
trasts with a tighter strip defined by colour excesses tied to space reddenings and reddening-independent indices (Turner 1995).

Incidentally, luminosities derived for cluster Cepheids do not depend upon $R$, the ratio of total to selective extinction. Cluster distances do, but luminosities for member Cepheids are tied only to luminosities inferred for similarly-reddened cluster ZAMS members, which are independent of $R$. The scatter in ZAMS-fitted absolute magnitudes varies from $5 \times$ to $13 \times$ the scatter in $(B-V)_{0}$ for mainsequence $B$ and A stars, so uncertainties of \pm 0.01 in $(B-V)_{o}$ for careful cluster studies translate into uncertainties of \pm 0.05 to \pm 0.13 in $M_{V}$ for individual ZAMS stars; samples of 15 or more ZAMS stars reduce the scatter in mean cluster distance moduli to less than \pm 0.03 ( $\sim 1 \%$ in distance). Statements that cluster distance moduli cannot be determined to better than \pm 0.2 ( $9 \%$ in distance) are therefore not realistic appraisals of the current state of the art.

\section{Association Cepheids}

While a number of long period Cepheids are coincident with $O B$ associations, main-sequence companions of 20-day Cepheids must have spectral types later than B2; such Cepheids cannot be physically related to the youngest association subgroups. Membership in older subgroups of associations is possible, but such subgroups are more spatially dispersed and more challenging to study than open clusters. Yet positive results have come from such studies, including the detection of a previously unrecognised cluster containing the long period Cepheid WZ Sgr. For distances in excess of $2 \mathrm{kpc}$ the dispersion in distance modulus expected from line-of-sight distance spread in associations is less than \pm 0.06 (Turner 1988), so association Cepheids can be reliable calibrators.

\section{The ZAMS calibration}

The standard ZAMS is tied to the distance of the Hyades cluster. The shape at the faint end is defined by the main sequence for Praesepe, the zero-point is established by the Hyades, and a normalisation to solar metallicity is made using Pleiades stars. Van Leeuwen's (1983) determination of the Pleiades distance using spectroscopic parallaxes is an example of various techniques also used to derive the Hyades distance, but cannot replace the Hyades calibration. Spectroscopic parallaxes use trigonometric parallaxes for nearby stars, and are just as susceptible to systematic error as more direct techniques. The zero-point uncertainty is still a problem to be addressed.

\section{References}

Fernie, J. D. 1990a, ApJS, 70, 153

Fernie, J. D. 1990b, ApJ, 354, 295

Gieren, W. P., \& Fouqué, P. 1993, IAU Coll., 139, 72

Turner, D. G. 1988, ASP Conf. Ser., 4, 178

Turner, D. G. 1995, in preparation

van Leeuwen, F. 1983, Ph.D. Thesis, Leiden 